\title{
Evaluation Of The Efficacy Of Fluorescent Staining And Chicago Sky Blue Staining As Methods For Diagnosis Of Dermatophytosis In Hair And Nails
}

This article was published in the following Dove Press journal: Clinical, Cosmetic and Investigational Dermatology

\author{
Basma Mourad' \\ Mayada Ismail' \\ Soha Hawwam' \\ Mina Msseha ${ }^{2}$ \\ Reham Hassan ${ }^{3}$ \\ 'Department of Dermatology and \\ Venereology, Faculty of Medicine, Tanta \\ University, Tanta, Egypt; ${ }^{2}$ Department of \\ Microbiology and Immunology, Faculty of \\ Medicine, Tanta University, Tanta, Egypt; \\ ${ }^{3}$ Department of Dermatology and \\ Venerelogy, Kafr Elsheikh General \\ Hospital, Ministry of Health, Kafr \\ Elsheikh, Egypt
}

\begin{abstract}
Purpose: Dermatophytes are fungi that cause infections affecting hair, nail, and skin; in nails they cause onychomycosis, while in hair they lead to tinea capitis. Detection of dermatophytes using traditional methods, including potassium hydroxide $(\mathrm{KOH})$ and culture on agar-based media leads to high rates of false-negative results. Here, we investigated more accurate diagnostic techniques, including Chicago sky blue staining and Calcofluor white fluorescent staining and compared them with traditional $\mathrm{KOH}$ and culture methods for the diagnosis of fungi causing onychomycosis and tinea capitis.
\end{abstract}

Patients and methods: This study was conducted using samples from 50 patients with dermatophytosis of the hair or nail. Samples were subjected to all the following laboratory investigations: $\mathrm{KOH}$ wet mount, culture on Sabouraud's dextrose agar, Chicago sky blue staining, and Calcofluor white staining. The results of the new diagnostic techniques were compared with those of the traditional methods.

Results: Calcofluor white stain and Chicago sky blue stain for dermatophytosis of hair and nail are more specific and sensitive as compared to traditional diagnostic methods. $\mathrm{KOH}$ wet mount is simple, rapid, and inexpensive test but lacks color contrast and gave more false positive (artifacts) and false-negative results as compared to these new stain methods.

Conclusion: Chicago sky blue and Calcofluor white staining are excellent methods for diagnosis of fungal infections, including those that cannot be confirmed using conventional methods.

Keywords: Calcofluor white stain, dermatophytes, onychomycosis, tinea capitis

\section{Introduction}

Dermatophytes are fungi that lead to infections of the hair, nails, and skin; in nails, they cause onychomycosis, while in hair they lead to tinea capitis. Onychomycosis is a common nail problem, accounting for up to half of all nail diseases and tinea capitis is the most relevant dermatophyte infection in children between 3 and 8 years of age. ${ }^{1}$

Traditional laboratory methods for diagnosis of dermatophytes, including microscopic observation following potassium hydroxide wet mounting and culture methods using agar-based media, have a high rate of false-negative results. ${ }^{2}$ Other techniques for the diagnosis of dermatophytosis have been investigated, including Chicago sky blue and Calcofluor white fluorescent staining. ${ }^{3}$

Chicago sky blue is a contrast staining method that allows differentiation between hyphae and epithelial cells and is used together with $\mathrm{KOH}$ as a clearing agent. Fungal filaments stain a distinct blue against a pale or purple background. ${ }^{4}$
Correspondence: Soha Hawwam Faculty of Medicine, Tanta University, El Geish Street, Tanta 3IIII, Egypt Tel +20 40966540238619

Email soha_abdallah80@yahoo.com 
The fluorescent stain, Calcofluor white, is used for the diagnosis of some parasitic organisms. It is a non-specific fluorochrome that binds to the cellulose and chitin contained in the cell walls of cellulose-containing organisms. This stain can be mixed with potassium hydroxide to clear the specimen and facilitate visualization of pathogenic elements. ${ }^{4}$

The aims of this study were to determine the utility of diagnostic microscopy methods, including Chicago sky blue and Calcofluor white fluorescent staining, and compare them with traditional $\mathrm{KOH}$ wet mounting and culture techniques for the diagnosis of fungi causing onychomycosis and tinea capitis.

\section{Patients, Materials, And Methods Patients}

Fifty patients participated in the present study: 25 with tinea capitis, comprising 20 females and five males, aged from 2 to 10 years; and 25 with onychomycosis, comprising 2 males and 23 females, aged from 23 to 60 years. Patients were recruited from the outpatient clinic of the Dermatology Department, Tanta University Hospital. Written informed consent was obtained from the legal guardian of patients under 18 years of age and from all other patients themselves. The study was approved by the Ethics Committee of the Faculty of Medicine, Tanta University.

\section{Methodology}

All patients underwent a full clinical and dermatological examination and specimens were collected, along with a complete history. All samples were subjected to $\mathrm{KOH}$ mounting, Calcofluor white staining, Chicago sky blue staining, and fungal culture.

\section{Sample Collection}

Nail samples

Affected finger nails with onychomycosis were cleaned with $80 \%$ alcohol to remove contaminants. Cleaned nails were clipped short with nail clippers and scrapings collected from the nail bed and the undersurface of the nail plate using the blunt edge of a scalpel blade.

\section{Hair samples}

Approximately 2-10 hair roots were collected using epilation tweezers. Scrapings were collected from lesions using the blunt edge of a scalpel blade or glass slide. Specimens were kept in clean, separate containers.

\section{Staining Procedures \\ $\mathrm{KOH}$ Wet Mount}

One drop by a plastic Pasteur pipette of $20 \% \mathrm{KOH}$ was added to each specimen mounted on a slide and covered with a cover slip. Then, specimens were examined by light microscopy at $\times 100$ magnification to locate fungal elements and at $\times 400$ magnification to identify spores and hyphae.

\section{Chicago Sky Blue Stain (Sigma-Aldrich)}

Chicago sky blue stain is a contrast stain. One drop of $1 \%$ Chicago sky blue stain and one drop of $10 \% \mathrm{KOH}$ as a clearing agent were added to specimens mounted on slides and covered with a coverslip. All slides were placed in a humidifying chamber for 30 mins and then examined by light microscopy at $\times 100$ and $\times 400$ magnification.

\section{Calcofluor White Stain (Sigma-Aldrich)}

One drop of Calcofluor white stain, comprising $1 \mathrm{~g} / \mathrm{L}$ Calcofluor white M2R and Evans blue (Sigma- Aldrich) was added to each specimen, mounted on a slide. Slides were then left to stand for 10 mins and examined under fluorescence microscopy using blue light excitation (300-400 nm), with an emission wavelength with of approximately $355 \mathrm{~nm}$.

\section{Fungal Culture}

Specimens (either hair or nail) were collected in sterile containers (Petri dishes and tubes) labeled with a patient identification code and the date of inoculation. Each clinical specimen was cultured on Sabouraud's dextrose agar (SDA) with chloramphenicol and cycloheximide for primary isolation of dermatophytes and incubated at $37^{\circ} \mathrm{C}$ for 3 weeks. Two tubes of SDA containing chloramphenicol were used for identification of other fungal species, with material directly inoculated onto the surface of the media and incubated aerobically at $25^{\circ} \mathrm{C}$ and $37^{\circ} \mathrm{C}$ for 3 weeks. Plates were examined daily during the first week and twice weekly during the subsequent 2 weeks. Isolates were identified using standard laboratory procedures.

\section{Statistical Analysis}

Collected data were organized, tabulated, and statistically analyzed using SPSS software (Statistical Package for the Social sciences, version 20 SPSS Inc. Chicago, IL, USA). The chi-square test was used to evaluate significance and $\mathrm{P}$-values $<0.05$ considered significant. 


\section{Results}

\section{Rates Of Fungus Detection Using Different Methods}

Fungal elements (hyphae and spores) were positive by potassium hydroxide wet mount in 16 patients (64\%) with onychomycosis. Further, results were positive for hair samples from 17 patients (68\%) with tinea capitis (Figure $1 \mathrm{~A}$ and $\mathrm{B}$ ).

Using Chicago sky blue staining 19 patients (76\%) were positive for fungal hyphae, which stained blue against a purple background. While with in tinea capitis 20 patients $(80 \%)$ showed positive results (Figure 1C and D).

Fluorescent microscopy showed that 21 patients with onychomycosis $(84 \%)$ were positive (showed clear fluorescent fungal hyphae against darker background) by Calcofluor white staining. Similarly, 22 patients with tinea capitis $(88 \%)$ were positive using this technique (Figure 2A and B).
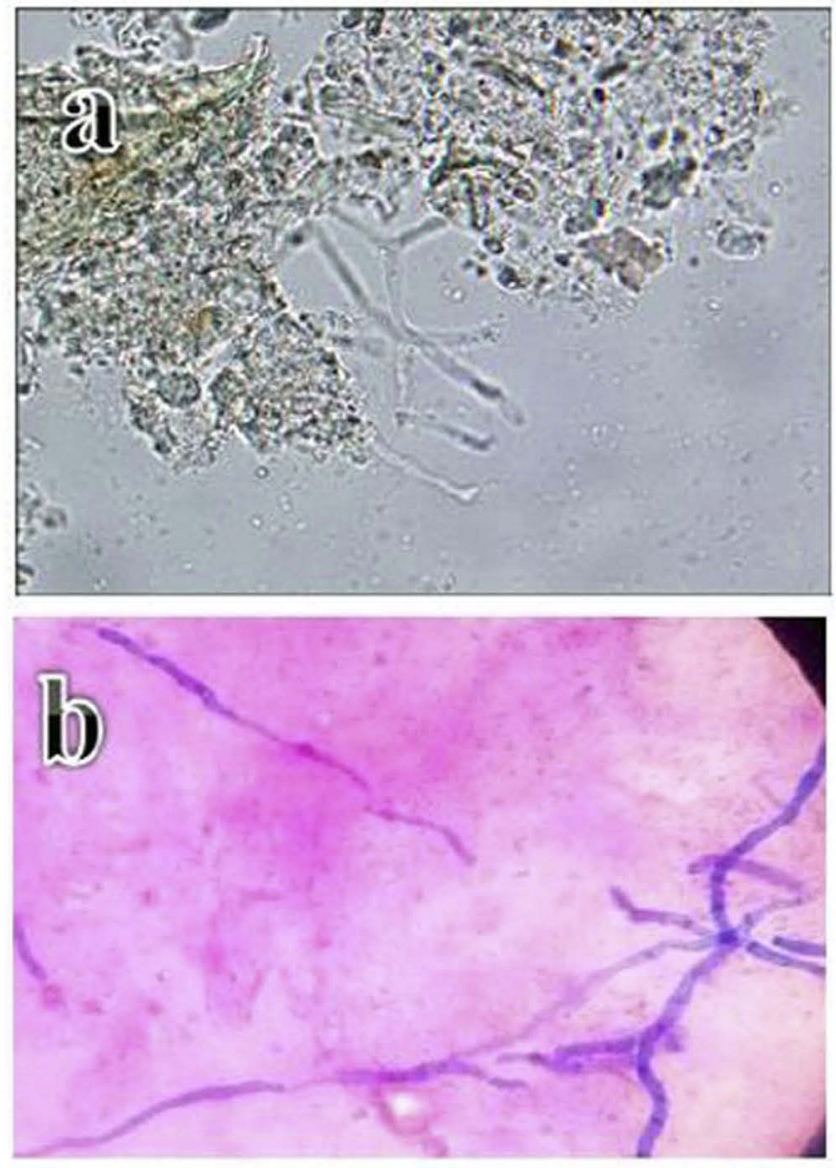
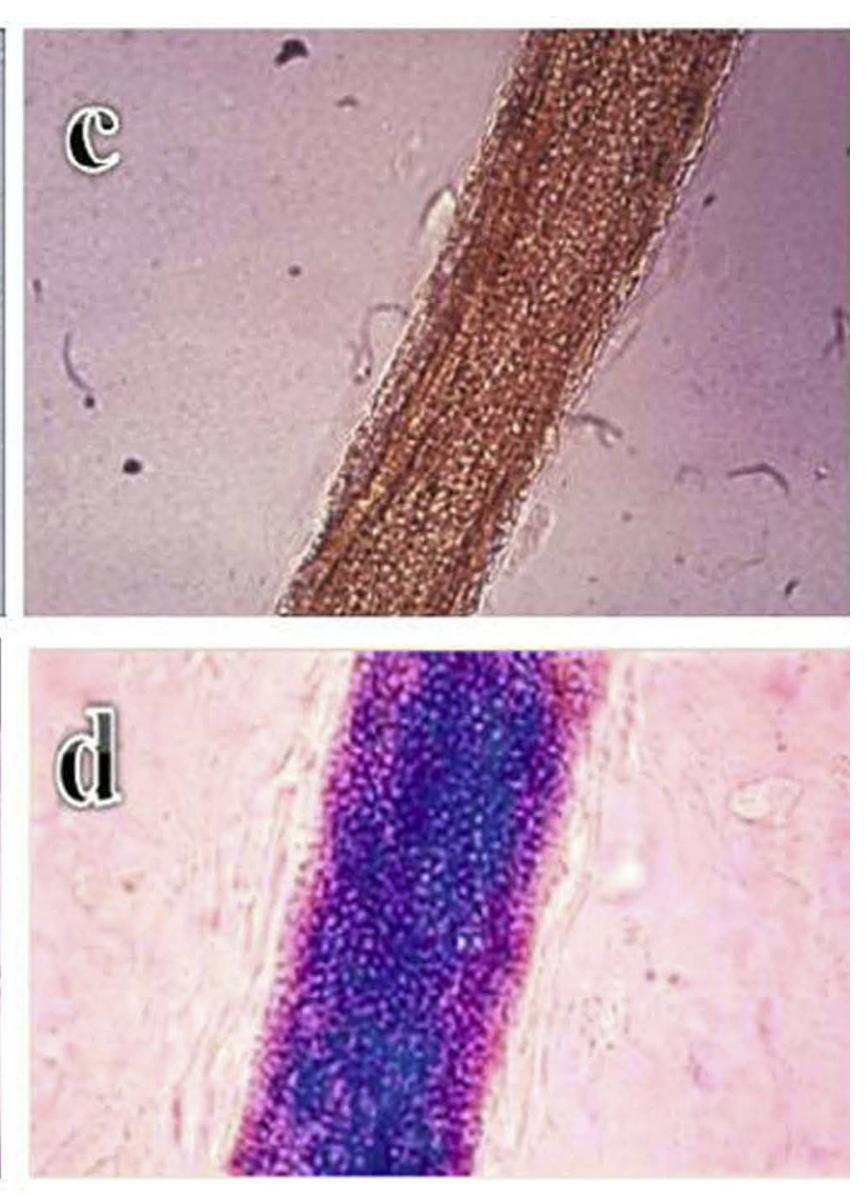

SDA cultures were positive in 15 patients $(60 \%)$ with onychomycosis while $17(68 \%)$ of those with tinea capitis were positive (Figure 2C-E).

Next, we calculated sensitivity, specificity, positive predictive, negative predictive, and accuracy values for each test in comparison with $\mathrm{KOH}$ wet mount and culture on SDA.

\section{Comparison Of Onychomycosis Detection Using Different Methods}

Compared with the results of traditional $\mathrm{KOH}$ wet mount, Chicago sky blue and Calcofluor white staining showed significantly higher sensitivity, specificity, positive predictive value, negative predictive value, and accuracy, with very low rates of false-positive and false-negative results. Further, in comparison with culture on SDA, Chicago sky blue stain showed non-significant differences in positive predictive, negative predictive, and accuracy values, with

Figure I Microscope images of: (A) translucent, non-pigmented, septate hyaline hyphae of Trichophyton rubrum (blue arrow) in a nail sample (I0\% $\mathrm{KOH}$ stain, $\times 400)$. (B) Endothrix hair invasion, with translucent and non-pigmented spores of Trichophyton violaceum (10\% $\mathrm{KOH}$ stain, $\times 100)$. (C) Bluish, narrow septate hyphae of Trichophyton rubrum against a pink background in a nail sample (Chicago sky blue stain, $\times 400$ ). (D) Endothrix hair invasion with blue spores of Trichophyton violaceum (Chicago sky blue stain, $\times 100)$. 

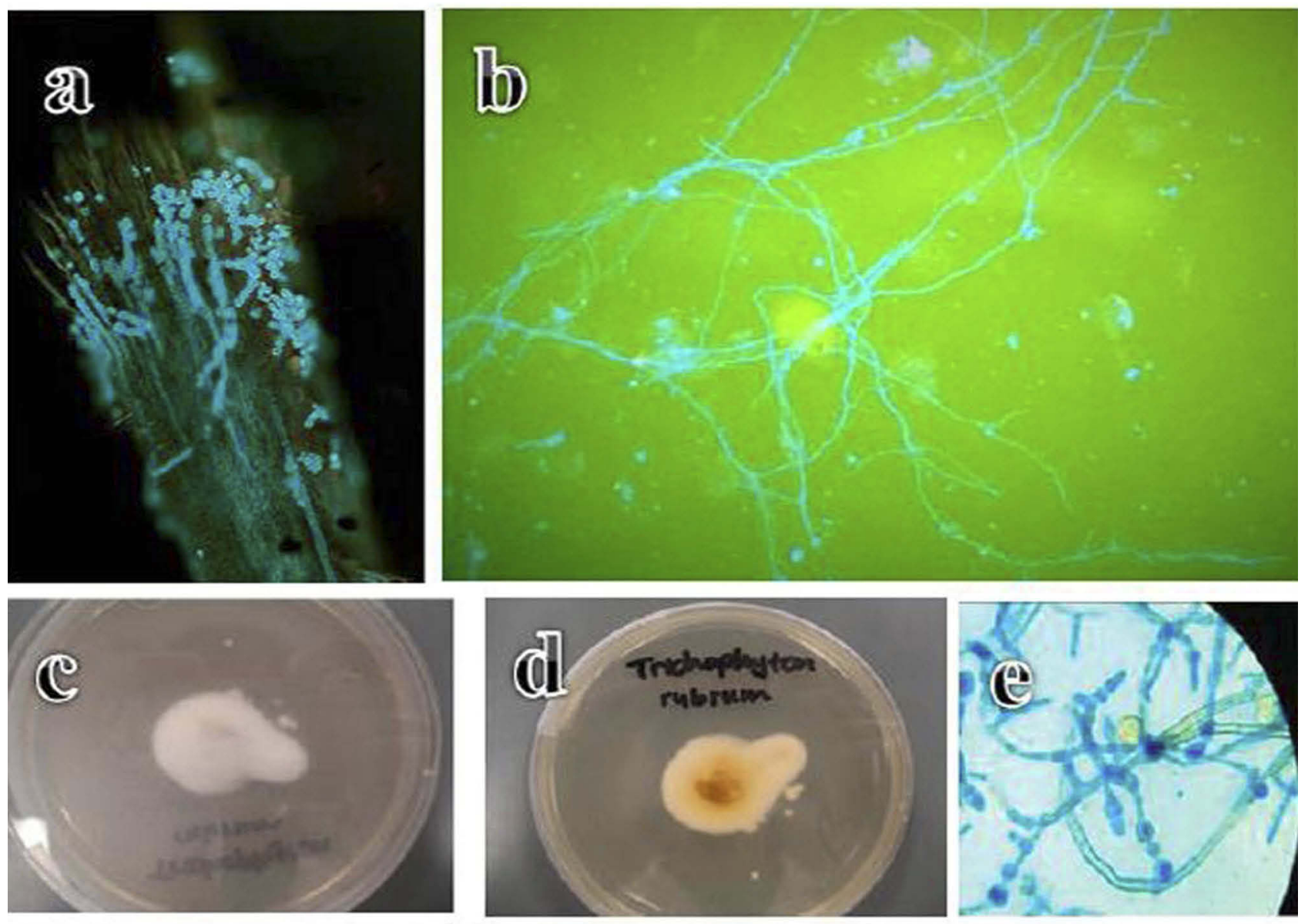

Figure 2 (A) Endothrix hair invasion with green, fluorescent hyphae and Trichophyton violaceum spores (Calcoflour white stain, fluorescent microscope, $\times 400$ ). (B) Fluorescent septate hyphae of Trichophyton rubrum against a darker background in a nail sample (Calcoflour white stain, fluorescent microscope, $\times 100)$. (C) Anterior surface of a Trichophyton rubrum culture. (D) Reverse surface of a Trichophyton rubrum culture. (E) Microscope image of blue, thin walled Trichophyton rubrum macroconidia showing the clavate shape and multiseptate, smooth walled structure (lacto phenol cotton blue stain, $\times 100$ ).

lower rates of false- positive and false-negative results. Moreover, Calcofluor white stain exhibited significantly higher sensitivity, specificity, positive predictive, negative predictive, and accuracy values than SDA culture. These results demonstrate that these two new staining methods are more effective for detection and diagnosis of fungal elements in patients with onychomycosis than traditional approaches (Table 1).

\section{Comparison Of Tinea Capitis Detection Using Different Methods}

Both Chicago sky blue and Calcofluor white staining exhibited significantly higher sensitivity, specificity, positive predictive, negative predictive, and accuracy values, with lower false-positive and false-negative results, relative to traditional $\mathrm{KOH}$ mounting. Further, comparison of Chicago sky blue stain with culture on SDA revealed non-significant differences in sensitivity, specificity, positive predictive value, negative predictive value, and accuracy. Calcofluor white stain had significantly higher sensitivity, specificity, positive predictive, negative predictive, and accuracy values than culture on SDA. These data indicate that Calcofluor white stain has higher efficacy and value for detection of fungal elements in patients with tinea capitis (Table 2).

\section{Results Of Mycological Culture}

In onychomycosis, Trichophyton rubrum was the most common dermatophyte species isolated (80\%), followed by Trichophyton mentagrophyte (48\%), Candida (40\%), and mixed infection (32\%).

In tinea capitis, Microsporum canis was the most common dermatophyte species isolated (80\%), followed by Microsporum audouinii (56\%), Trichophyton violaceum (40\%), and mixed infection (24\%). 

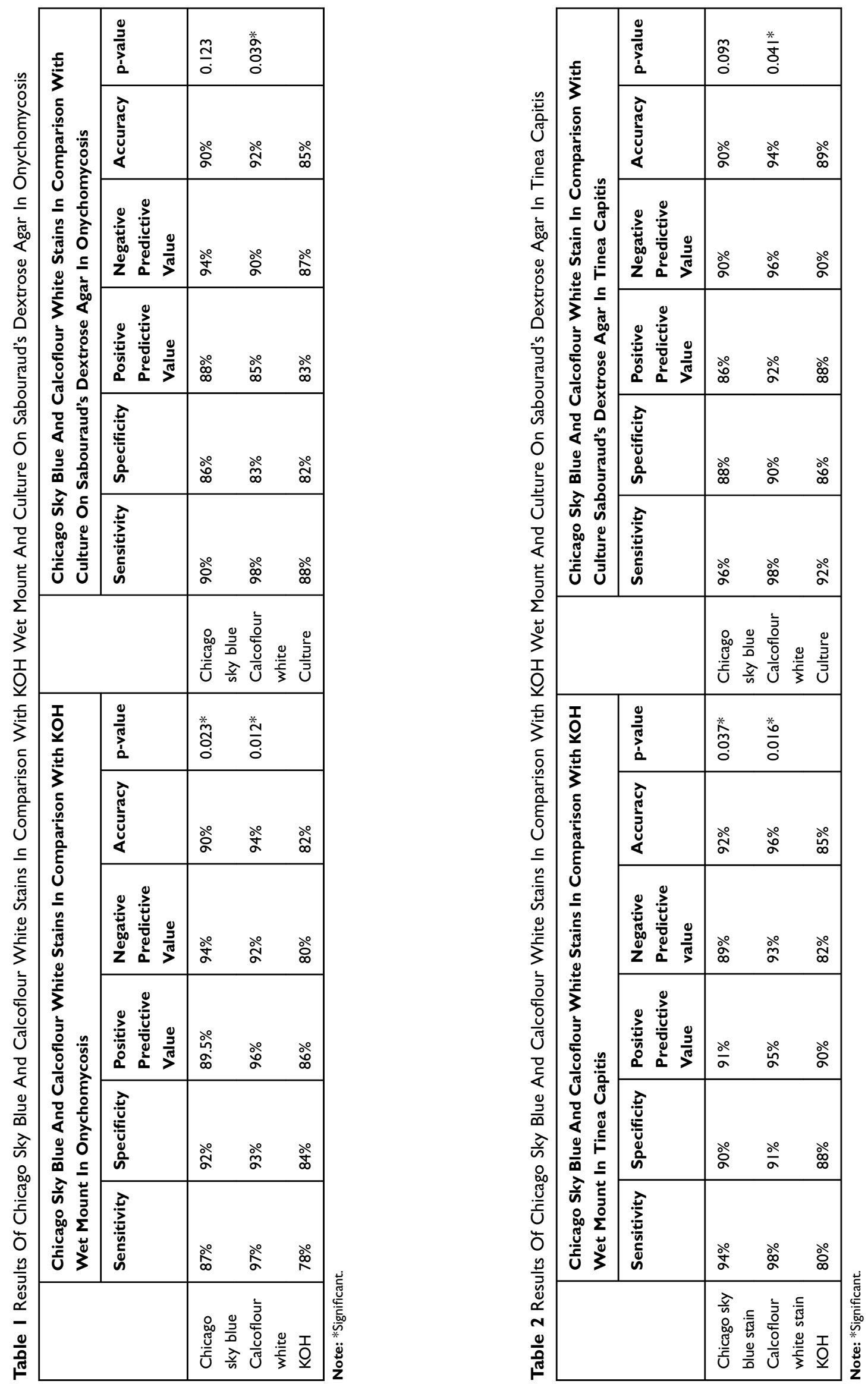


\section{Discussion}

Dermatophytes are group of fungi that grow on skin, hair, and nail, causing dermatophytosis or superficial mycosis. They are generally classified into three genera, Epidermophyton, Trichophyton, and Microsporum. ${ }^{1}$

Tinea capitis is the most common pediatric condition caused by dermatophyte infection worldwide. Common dermatophytes that cause tinea capitis include M. canis, M. audouinii, and Trichophyton tonsurans. ${ }^{12}$ Generally, tissue invasion is confined to the cutaneous layer, because of the inability of the fungi to penetrate deeper tissues; however, occasionally, subcutaneous invasion occurs and may manifest as a kerion. ${ }^{5}$

A high rate of false-negative results for dermatophyte detection are observed using traditional laboratory methods, including microscopic observation by potassium hydroxide and culture methods using agar-based media. ${ }^{4}$

Chicago sky blue stain is a contrast stain that can help to distinguish between hyphae and epithelial cells and is used together with $\mathrm{KOH}$ as a clearing agent. When stained, fungal filaments appear distinct blue against a pale or purple background. ${ }^{6}$

Calcofluor white stain is a non-specific fluorochrome that binds with cellulose and chitin contained in the cell walls of cellulose-containing organisms and facilitates the visualization of pathogenic elements. ${ }^{7}$

In the present study, using $\mathrm{KOH}$ wet mount, $64 \%$ of samples from patients with onychomycosis and $68 \%$ of those from tinea capitis were positive for fungal elements (hyphae or spores). These results are consistent with other studies, ${ }^{8,9}$ that reported rates of positivity of $62 \%$ and $66 \%$, respectively, for fungal elements (hyphae and/or spores) in patients with onychomycosis.

Here, we compared the results of Chicago sky blue staining with those from traditional $\mathrm{KOH}$ wet mount. In onychomycosis, $\mathrm{KOH}$ wet mount results were positive for $64 \%$ of samples, while Chicago sky blue stain positively detected fungal elements in $76 \%$. Further, Chicago sky blue staining exhibited significantly higher sensitivity, specificity, positive predictive value, negative predictive value, and accuracy and lower levels of false-positive results, relative to $\mathrm{KOH}$ wet mount. In tinea capitis, Chicago sky blue stain also resulted in significantly higher sensitivity, specificity, positive predictive value, negative predictive value, and accuracy with lower rates of false-positive and false-negative results than traditional $\mathrm{KOH}$ wet mount.
These results reflect that, while the standard $\mathrm{KOH}$ mount technique is rapid and inexpensive, it does not produce a color contrast and requires relatively high skill levels for smear interpretation, as well as being time consuming and potentially leading to false-negative reports in samples with sparse fungal elements. Using Chicago sky blue stain, dermatophytes were detected easily at $\times 100$ magnification as blue stained fungal hyphae against the purple to pink cellular background and were confirmed by observation at $\times 400$ magnification to visualize septate filaments. This technique also highlights the morphological characteristics of both spores and hyphae and the color contrast facilitates fungal element identification, even in samples where they are sparse.

The findings presented by Lim and Lim $(2008)^{4}$ agreed with the results reported here; they reported that Chicago sky blue stain had $100 \%$ sensitivity after 5 mins when examined under the microscope and was more sensitive and more specific than $\mathrm{KOH}$ preparation for the diagnosis of hair and nail dermatophytes. The results of the present study are also consistent with those of Shwetha et al $(2017)^{10}$ who reported that Chicago sky blue stain was $100 \%$ sensitive for detection of dermatomycosis and $83.4 \%$ sensitive for diagnosis of onychomycosis, whereas $\mathrm{KOH}$ mount was only $93 \%$ and $66.7 \%$ sensitive for detection of dermatomycosis and onychomycosis, respectively. Moreover, Chicago sky blue stain exhibited $100 \%$ specificity for detection of dermatomycosis and onychomycosis, whereas $\mathrm{KOH}$ mount was only $77 \%$ specific.

In the present study, the results for diagnosis of onychomycosis by culture were positive, but less effective than those obtained by Chicago sky blue staining, with some false-positive results. The results for diagnosis of tinea capitis were also positive by culture, but the method was less effective than Chicago sky blue staining, which had a low rate of false-positive results. Overall, the results using Chicago sky blue stain were excellent, emphasizing the superiority of this technique over SDA culture.

The results of Bonifaz et al (2013) ${ }^{11}$ agreed with those presented here; they reported that Chicago sky blue stain had high sensitivity $(100 \%)$ vs culture $(60.9 \%)$ and was clearly superior to culture for the rapid diagnosis of dermatophytosis of the hair or nails.

In the present study, we also used Calcofluor white stain for diagnosis of fungal hyphae by fluorescence microscopy and compared the results with those of $\mathrm{KOH}$ wet mount and SDA culture. Calcofluor white stain produced excellent results, with $84 \%$ and $88 \%$ positive rates 
for diagnosis of onychomycosis and tinea capitis, respectively. Bright fungal elements were visible on examination of positive samples by fluorescence microscopy. The results of $\mathrm{KOH}$ wet mount analysis of the same samples showed that $64 \%$ and $68 \%$ were positive for onychomycosis and tinea capitis, respectively, indicating that Calcofluor white stain had superior sensitivity to $\mathrm{KOH}$ wet mount alone for the diagnosis of fungi causing tinea capitis and onychomycosis.

Comparison of Calcofluor white stain with $\mathrm{KOH}$ wet mount results in onychomycosis demonstrated that Calcofluor white stain generated fewer false-positive results (20\%), with a significantly higher specificity, sensitivity, and accuracy. Similarly, in tinea capitis, Calcofluor white staining resulted in low rates of false-positive results, with significantly higher specificity, sensitivity, and accuracy than $\mathrm{KOH}$ wet mounting.

Our results were consistent with those of Attal et al (2015), ${ }^{12}$ who demonstrated that Calcofluor white stain had higher sensitivity than $\mathrm{KOH}$ in direct examination for dermatophytes of hair and nail. Further, Dass et al $(2015)^{7}$ reported that Calcofluor white stain was an excellent method for detection of fungal agents in cases with clinically suspected onychomycosis and that this method had higher sensitivity than $\mathrm{KOH}$.

Shwetha et al (2017) ${ }^{10}$ compared Chicago sky blue stain with $\mathrm{KOH}$, using Calcofluor white stain as the gold standard, in respect of sensitivity, specificity, positive and negative predictive values, diagnostic efficacy, and cost-effectiveness. They concluded that Calcofluor white stain is highly effective, but noted that it had the disadvantage of requiring a fluorescence microscope, which is relatively expensive. Chicago sky blue stain is equally effective, but without the cost burden associated with the Calcofluor white staining method.

Comparison between the results obtained using Calcofluor white stain and those from SDA culture revealed that the Calcofluor white method had significantly higher sensitivity, specificity, positive predictive value, negative predictive value, and accuracy, with fewer false-positive results, in onychomycosis. While in tinea capitis Calcofluor white stain showed significantly higher sensitivity, specificity, positive predictive value, negative predictive value, and accuracy. These data demonstrate that the mycological culture is less sensitive than Calcofluor white stain for diagnosis of dermatophytosis of hair and nails.
Dass et al $(2015)^{7}$ reported that, although culture is a good method for definite diagnosis of dermatophytes, it generates numerous false-negative results, with positive results in only (39\%), while Jeelani et al $(2016)^{8}$ reported that culture generated positive results in $58 \%$ of dermatophytes in onychomycosis.

In the current study among the various dermatophytes isolated from patients with onychomycosis, T. rubrum was the most common, followed by $T$. mentagrophyte, Candida, and mixed infections. While in patients with tinea capitis, M. canis was the most common dermatophyte species, followed by $M$. audouinii, T. violaceum, and mixed infection. These results are consistent with other studies, which found that T. rubrum and T. mentagrophyte were the most common pathogens among patients with onychomycosis, as well as the agent most frequent causes of skin dermatophytosis. ${ }^{12,13}$

\section{Conclusions}

From the results of the present study, we conclude that Calcofluor white and Chicago sky blue staining are more specific and sensitive for detection of dermatophytosis of hair and nail than traditional diagnostic methods. $\mathrm{KOH}$ wet mount is a simple, rapid, and inexpensive test, but lacks color contrast and generates more false-positive (artifacts) and false-negative results relative to these new staining methods.

\section{Disclosure}

The authors report no conflicts of interest in this work.

\section{References}

1. Nenoff P, Kruger C, Schaller J, et al. Mycology-an update part 2: dermatomycoses, clinical picture and diagnostics. $J$ Dtsch Dermatol Ges. 2014;12(9):749-777.

2. Gupta AK, Zaman M, Singh J. Diagnosis of Trichophyton rubrum from onychomycotic nail samples using polymerase chain reaction and calcofluor white microscopy. J Am Podiatr Med Assoc. 2008;98 (3):224-228. doi:10.7547/0980224

3. Chander G, Khurana A. Onychomycosis, newer insights in pathogenesis and diagnosis. Indian J Dermatol Venereol Leprol. 2012;78 (3):263-270. doi:10.4103/0378-6323.95440

4. Lim SL, Lim CS. New contrast stain for the rapid diagnosis of pityriasis versicolor. Arch Dermatol. 2008;144(8):1058-1059.

5. Shy RR. Tinea corporis and tinea capitis. Pediatr Rev. 2007;28 (5):164-174. doi:10.1542/pir.28-5-164

6. Lodha N, Poojary SA. A novel contrast stain for the rapid diagnosis of pityriasis versicolor: a comparison of Chicago sky blue 6B stain, potassium hydroxide mount and culture. Indian J Dermatol. 2015;60 (4):340-344. doi:10.4103/0019-5154.160476

7. Dass M, Vijayraj EV, Pavavni K, et al. Comparison of $\mathrm{KOH}$, Calcofluor white and fungal culture for diagnosing fungal onychomycosis in an urban teaching hospital, Hyderabad. Indian J Microbiol Res. 2015;2(3):148-153. doi:10.5958/2394-5478.2015.00004.7 
8. Jeelani S, Lanker AM, Jeelani N, et al. Clinico-mycological study of onychomycosis in children from a tertiary care center. Indian J Pediatr Dermatol. 2016;17(2):95-100. doi:10.4103/2319-7250.179411

9. Dogo J, Afegbua SL, Dung EC. Prevalence of tinea capitis among school children in Nok community of Kaduna State, Nigeria. $J$ Pathogens. 2016;8:1155.

10. Shwetha JV, Harsha TR, Shariq AK, et al. Comparison of Chicago sky blue (novel stain) with Calcofluor white and potassium hydroxide mount for rapid diagnosis of dermatomycosis and onychomycosis in a tertiary care centre. Int J Curr Res. 2017;9(2):46864-46868.
11. Bonifaz A, Rios-Yuil JM, Arenas R, et al. Comparison of direct microscopy, culture and calcofluor white for the diagnosis of onychomycosis. Revista Iberoam Micol. 2013;30(2):109-111. doi:10.10 16/j.riam.2012.07.001

12. Attal RO, Chaudhary RA, Deotale VS, et al. A clinicomycological study of onychomycosis in a rural hospital in central India. Int J Res Med Sci. 2015;3(5):1131-1137. doi:10.5455/2320-6012.ijrms20150519

13. Martinez DA, Oliver BG, Gräser Y, et al. Comparative genome analysis of Trichophyton rubrum and related dermatophytes reveals candidate genes involved in infection. M Bio. 2012;3(5):e00259-272.

\section{Publish your work in this journal}

Clinical, Cosmetic and Investigational Dermatology is an international, peer-reviewed, open access, online journal that focuses on the latest clinical and experimental research in all aspects of skin disease and cosmetic interventions. This journal is indexed on CAS.
The manuscript management system is completely online and includes a very quick and fair peer-review system, which is all easy to use. Visit http://www.dovepress.com/testimonials.php to read real quotes from published authors. 